\title{
An Adaptive Gravitational Search Routing Algorithm for Channel Assignment based on WSN
}

\author{
Sachi Pandey ${ }^{1 *}$ and R. P. Mahapatra ${ }^{2}$ \\ Department of Computer Science and Engineering \\ SRM University, NCR Campus ${ }^{12}$ \\ sachipandey.231@gmail.com ${ }^{1}$, mahapatra.rp@gmail.com ${ }^{2}$
}

\begin{abstract}
A wireless sensor network is a wireless, ad hoc network, made of a ample number of nodes, whose positions occur randomly. WSN contains unique transducers with a communicative design for monitoring and recording conditions at remote locations. Generally measured parameters are temperature, stickiness, weight, wind course and speed, brightening force, vibration force, sound power, control line voltage, substance focuses and poison levels. Communication costs can be reduced by some algorithms by removing or reducing nodes redundant sensor information, avoid forwarding data that is of no use. As nodes can filter the data they forward, they can measure averages or direction. As the sensors work remotely, energy consumption related issues are considered as the critical parameters. The vitality utilization rates for sensors in a remote sensor organize changes significantly in light of the conventions the sensors use for correspondences. In this paper, a new algorithm is designed to integrate energy consumption and hops into routing choice. The adaptive GSA method will increase the efficiency of the system, obtains balanced transmission among the nodes and reduce the energy consumption of the routing, and extends the network lifetime.
\end{abstract}

Keywords: Sensor Networks, Gravitational search algorithm, PSO, AGSA, Energy Consumption

\section{Introduction}

During emergencies such as metrological natural hazards, floods and residential fires, infrastructure-based communication may not exist; therefore, the availability of alternative communication means is of utmost importance for delivering the disaster related information to concerned rescue workers. Recently, WSNs have gone beyond the fine-grained continuous monitoring platforms and became one of the enabling technologies for disaster management [1].The rapid evolution of wireless technologies and the significant growth of wireless network services have made wireless communications a ubiquitous means for transporting information across many different domains. Within the framework of Wireless Sensor Networks (WSNs), there are many potential possibilities where a WSN can be deployed to support numerous applications [2, 3]. A WSN is an infrastructure less network consisting of ten to some thousands autonomous low power sensors organized in an ad hoc manner. These sensors are able to gather and process information from an environment, and communicate with each other. WSNs consist of sensors placed in remote areas to collect data and send it back to a base station (BS). Different attributes can characterize the sensor nodes including size, battery consumption, and power level, lifetime of operation, movement characteristics, position characteristics, and failure characteristics [4]. The routing protocols that have been proposed for sensor networks can be broadly classified as flat and hierarchical protocols. Hierarchical protocols arrange the network nodes into a few sensible levels. According to protocol operation, these protocols can be classified as multipath-based, query-based, 
negotiation-based, QoS-based, or coherent-based routing techniques[5]. In multipathbased routing, multiple paths are maintained between a source-destination pair. In querybased routing, the destination node sends a query through the network and the node with this data, sends an answer. In arrangement based directing, abnormal state information descriptors are utilized to kill repetitive information transmissions through transaction. In QoS-based routing, certain QoS metrics have to be satisfied while routing data through the network. In coherent-based routing, sensors cooperate in processing data flooded throughout the network[6]. Contrary to mobile ad-hoc networks (MANETs), the wireless mesh backbone is usually stationary, and as opposed to wireless sensor networks (WSNs), there is no limitation on the nodes' power consumption. In WMN consists of set of static mesh routers .Using the static mesh routers, we extend the reach of precise internet gateway results for each mobile users in the WMN [7]. Multi-hop routes are dynamically formed by using static mesh routers among mobile client and the internet gateway An indispensable concern in WMNs, however, is to boost the physical layer capacity and to reduce interference, which is normally achieved by equipping each node with a limited number of radios, usually less than or equal to the number of available channels[8]. Interference arises whenever two or more nodes try to communication on the same channel within each other's reception range at the same time. It results in high packet loss rate, high delay and low network throughput. Channel assignment algorithms are proposed to solve this problem. The goals of channel assignment algorithm are minimizing the interference between communicating links of wireless network and ensuring the quality of communication [9].

\section{Related Work}

MelikeYigit et al. [10] have stated a Wireless Sensor Networks (WSNs) it was one of the most promising solutions for smart grid applications due to advantages, such as their low-cost, different functionalities, and successful adoption to smart grid environments. However, providing quality of service (QoS) requirements of smart grid applications with WSNs was difficult because of the power constraints of sensor nodes and harsh smart grid channel conditions, such as RF interference, noise, multi-path fading and node contentions. To address these communication challenges, link-quality-aware routing algorithm (LQ-CMST) as well as the priority and channel-aware multi-channel (PCAMC) scheduling algorithm had been used for smart grid applications. It significantly reduce communication delay and the choice of encoding and modulation schemes was critical to meet the requirements of envisioned smart grid applications

M. Jahanshahi et al. [11] has proposed a Multicast routing it generally an efficient mechanism for delivering identical content to a group of receivers. Multicast was also deemed a key enabling service for a wealth of audio and video applications as well as data dissemination protocols over the last-mile backhaul Internet connectivity provided by multi-channel multi-radio wireless mesh networks (MCMR WMNs). Major prior art multicast protocols in these networks centre around heuristic or meta-heuristic initiatives in which channel assignment and multicast routing were considered as two separate subproblems to be solved in sequence. Within this perspective, however, the interplay between the two sub-problems would essentially be ruled out from the computations, resulting in sub-optimal solutions for network configuration. It was targeted at promoting the adoption of cross-layer design for joint channel assignment and multicast tree construction problem in MCMR WMNs. In particular, a comprehensive crossoptimization framework based on the binary integer programming (BIP) formulation of the problem was presented which also addresses the hidden channel problem in MCMR WMNs. 
Xiao-Chen Hao et al. [12] has developed a wireless sensor network, communication interference problem it was serious due to the widespread use of wireless sensor network. Meanwhile, the network may perform abnormally for the low energy node failure. The energy consumption of data retransmission was the main reason for node failure. The data retransmission was caused by communication interference. As we all know, the multichannel technology was an effective way to alleviate the interference among nodes by using the available channel resource reasonably. It had higher complexity, because they assign channel for link through respectively determining the receiving and sending channel of two nodes on the link. However, the sensor node's energy and the ability of computing access were limited greatly. Furthermore, a kind of Energy Efficiency based multiple channels allocation algorithm was used. In addition, the algorithm only assigns receiving channel for sensor node to avoid the high complexity of algorithm.

\section{Problem Definition and Proposed Methodology}

The advances in wireless communication and electronics technologies, wireless sensors are getting smaller, cheaper, and more powerful. Due to the fast development of the microprocessor, sensor and transceiver, there is great applications foreground about WSNs. Also since we often use these networks in rough and in accessible environments such as battlefields, volcanoes, forests and so on, normally there is low possibility to change or recharge the defective or dead nodes. Hence, the main difference between WSNs and other classic wireless networks is that WSNs are hypersensitive and vulnerable to energy. Therefore, how to use the limit energy of WSNs to maximize the life of WSNs becomes the all-important problem of routing design. Most of the routing algorithms for sensor networks require location information for sensor nodes. As a rule, area data is required keeping in mind to calculate the distance between two particular nodes. so that energy consumption can be assessed. In this way, area data can be used in routing data in energy efficient way. When all the above-mentioned drawbacks are resolved in the literary works, the performance of our system could be improved. However, the lack of solution for such drawbacks has motivated us to do the research work in this area.

Reducing the energy consumption of network nodes is one of the most important problems for routing in wireless sensor networks because of the battery limitation in each sensor. In this paper, we have proposed a new Adaptive Gravitational Search Algorithm (AGSA) based routing technique that uses special parameters in its competency function for reducing energy consumption of network nodes. Hence, a new algorithm that is designed to integrate energy consumption and hops into routing choice. Therefore, the adaptive GSA method will increase the efficiency of the system, obtains balanced transmission among the nodes and reduce the energy consumption of the routing, and extends the network lifetime. The proposed technique will be implemented in MATLAB and the comparison will be added with existing technique to show the improvements of our proposed method.

\section{Block Diagram of Proposed Method}

The block diagram of the proposed method is depicted in the figure 1; it shows the components of the heterogeneous wireless sensor network. Components of the wireless sensor node: A wireless sensor network (WSN) is a wireless network consisting of spatially distributed independent devices using sensors to monitor environmental conditions. A WSN system employs a gateway that provides wireless connectivity back to the wired world and distributed nodes. A WSN node contains several technical components. These include:

Sensing Unit: - Sensing node is one of the main components of the wireless sensor node. It consists of two components sensor and Analog digital converter. This unit provides the 
information gathering capabilities from the surroundings, such as temperature, humidity, pressure. The analog signals produced by the sensor based on observed phenomena are converted into digital signal by ADC. Then this digital signal is fed to processing unit.

Processing unit: - Processing Unit is the controller of the wireless sensor node; every other component is managed through processing unit. Processing unit consists of controller and memories integrated into the embedded board. It manages the function of each component and provides a communication among them.

Transceiver unit: - This unit is responsible to provide communication link between two nodes. Transceiver unit follows process of converting stream of bits to be transmitted into the Radio frequency waves and recover them at the other end. The connectivity of the network is based on this unit of node.

Power unit: - One of the most important components of the node is power unit. Each component in the node is connected with the power unit to be in active mode. Mostly battery power is used, but other sources are also possible. Power generator can be used, where long network lifetime is required. Solar cells are used to generate power for outdoor applications. There are few other components which are embedded in the node like, GPS. Most of the sensor network application, sensing tasks, routing techniques required the knowledge physical location of the node. So, it is necessary to have GPS for location finding system equipped with the node.

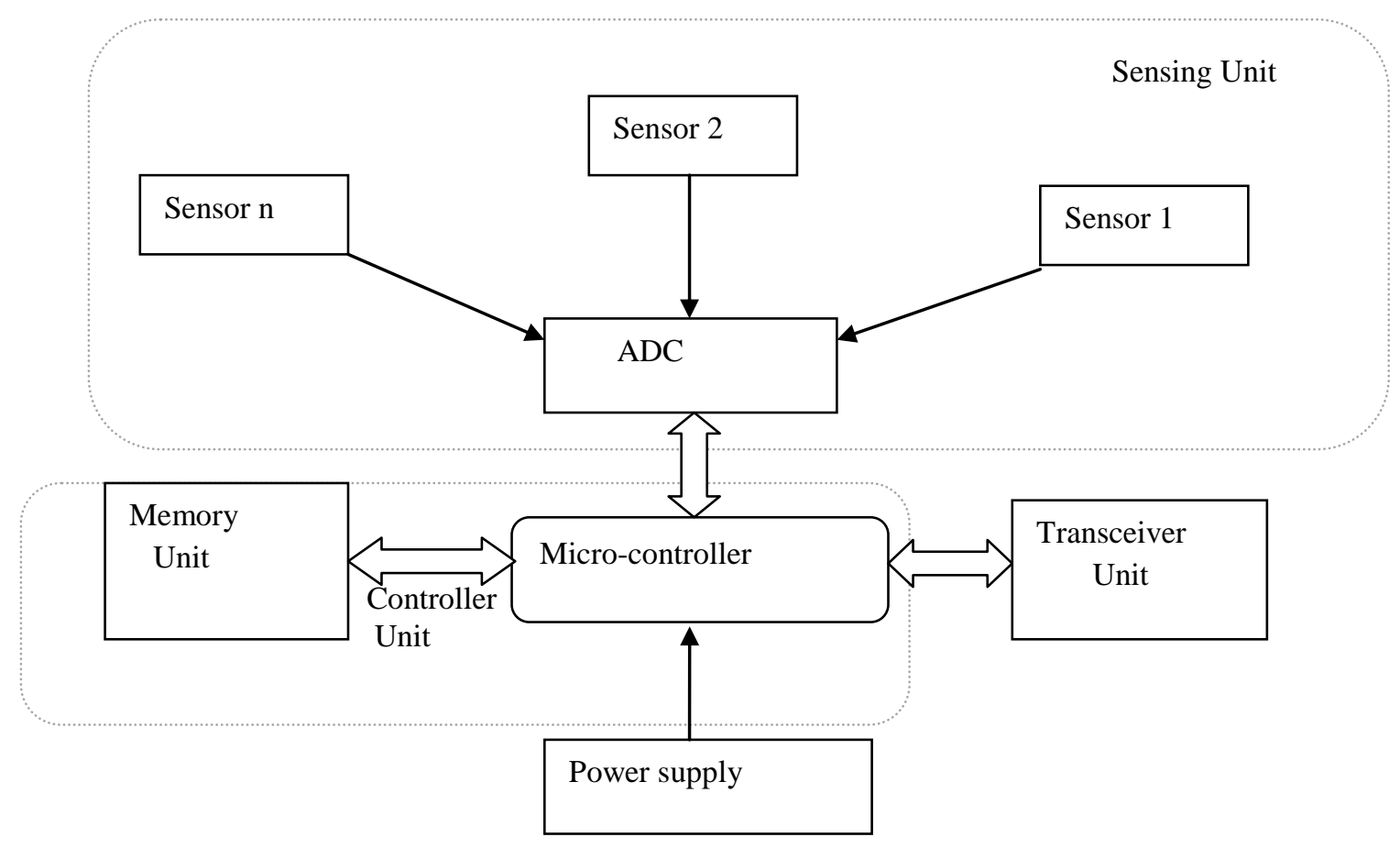

Figure 1. Heterogeneous Wireless Sensor Network

\section{Proposed Algorithm}

In this optimization two different algorithm approaches have been clubbed to achieve satisfied throughout in terms of battery lifetime of node and hop routing. AGSA optimization employs gravitational search algorithm and PSO. The flow of the optimization technique is depicted in figure 2 . 


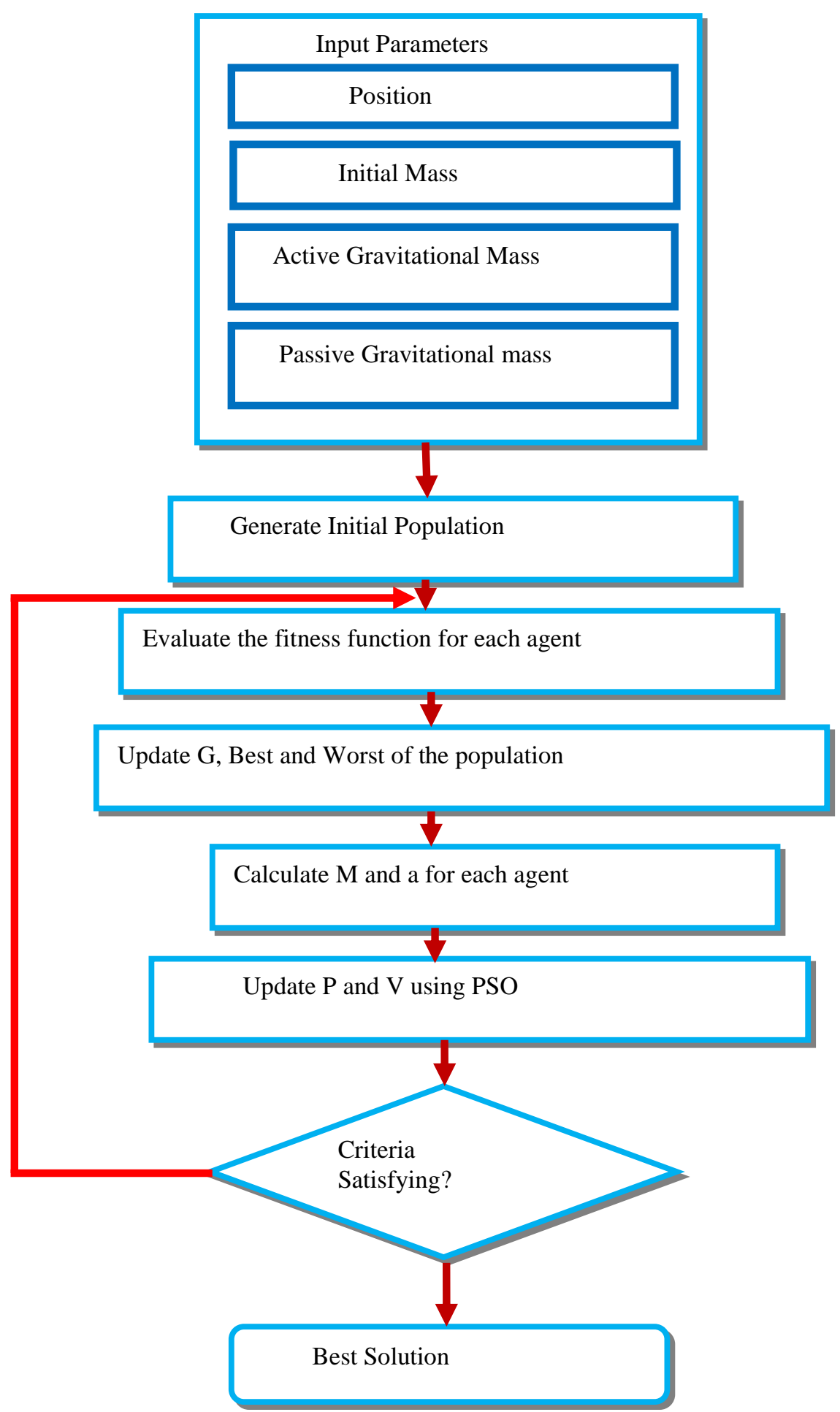

Figure 2. Flow of Proposed Optimization

Gravitational search algorithm has been inspired by Newtonian's law of Gravity and the law of Motion. The algorithm is proposed to improve the performance in the exploration and exploitation capabilities of a population based algorithm, based on gravity rules. PSO is motivated from the simulation of the social behavior (flock of birds). This optimization approach update the population of particles by applying an operator according to the fitness information obtained from the environment so that the individuals of the population can be expected to move towards the better solution. 
Law's of gravity: The gravitation is the ability of masses to accelerate toward each other. It is one of the fundamental interactions in nature. Every particle in the universe attracts every other particle. Gravity is everywhere. The inescapability of gravity makes it different from all other natural forces. Newton's law of gravity, each particle attracts every other particle with a gravitational force F. The gravitational force between two particles is directly proportional to the product of their masses $\mathbf{M}_{1}, \mathbf{M}_{2}$ and inversely proportional to the square of the distance $\mathrm{d}^{2}$ between them and $\mathrm{G}$ is the gravitational constant.

$$
F=G \frac{M_{1} \times M_{2}}{d^{2}}
$$

Newton's second law says that when a force, F, is applied to a particle, its acceleration, a, depends only on the force and its mass, $\mathrm{M}$.

$$
a=\frac{F}{M}
$$

Active gravitational mass, $M_{x}$, is a measure of the strength of the gravitational field due to a particular object. For example, the gravitational field that one experiences on the Moon is weaker than that of the Earth because the Moon has less active gravitational mass. Passive gravitational mass, $M_{y}$, is a measure of the strength of an object's interaction with the gravitational field. Inertial mass, $M_{i}$, is a measure of an object resistance to changing its state of motion when a force is applied. An object with large inertial mass changes its motion more slowly, and an object with small inertial mass changes it rapidly. Now, considering the above-mentioned aspects, we rewrite Newton's laws. The gravitational force, $F_{l m}$, that acts on mass $l$ by mass $m$, is proportional to the product of the active gravitational of mass $m$ and passive gravitational of mass $l$, and inversely proportional to the square distance between them. $a_{l}$ is proportional to $F_{l m}$ and inversely proportional to inertia mass of $i$. More precisely, we can rewrite equations. (1) and (2) as follows:

$$
\begin{aligned}
& F_{l m}=G \frac{M_{y l} \times M_{x m}}{d^{2}} \\
& \boldsymbol{a}_{l m}=\frac{F_{l m}}{M_{i l}}
\end{aligned}
$$

Where, $M_{y l}$ and $M_{x m}$ represent the active gravitational mass of particle $l$ and passive gravitational mass of particle $m$, respectively, and $M_{i l}$ represents the inertia mass of particlel. 
Adaptive Gravitational Search algorithm: The steps of Adaptive search Algorithm are given below:

Adaptive Gravitational Search algorithm

1-Initialise $Z$ number of agents randomly $P_{i}=\left(p_{i}^{1} \ldots ., p_{i}^{e} \ldots \ldots, p_{i}^{z}\right)$ for $i=1 \ldots \ldots . . Z, T$ iterations.

2-Evaluate the fitness equation.

3- Perform Minimization or Maximization problem for all agents.

Minimization problem:

$$
\begin{aligned}
\operatorname{best}(q) & =\min . \text { fit }_{k}(q) \\
\operatorname{worst}(q) & =\min . \operatorname{fit}_{k}(q)
\end{aligned}
$$

4- Calculate G: $\quad G(q)=G_{0} e^{\left(\frac{-\beta q}{T}\right)}$

5- Update G, Best and worst.

6-Calculate M and a. $m_{l}(q)=\frac{\operatorname{fit}_{l}(q)-\operatorname{worst}(q)}{\operatorname{best}(q)-\operatorname{worst}(q)}$ And $a_{i}^{d}=F_{i}^{d}(q) / M_{l i}(q)$

7- Update Position and velocity using PSO.

$p_{i}^{d}(q)=p_{i}^{d}(q)+p_{i}^{d}(q+1)$

$v_{i}^{d}(q+1)=W(q) v_{i}^{d}(q)+F_{1} R_{k 1}\left(\right.$ Pbest $\left._{i}^{d}-p_{i}^{d}(q)\right)+F_{1} R_{k 1}\left(\operatorname{Sbest}_{i}^{d}-p_{i}^{d}(q)\right)$

\section{8- Criteria check.}

9-Best solution

\section{Explanation of Algorithm}

Step 1: Agents initialization: The positions of the $Z$ number of agents are initialized randomly.

$P_{i}=\left(p_{i}^{1} \ldots . ., p_{i}^{e} \ldots \ldots, p_{i}^{z}\right)_{\text {For }} i=1 \ldots \ldots . . Z$

$p_{i}^{e}$ Represents position of $i^{\text {th }}$ agent in $e^{\text {th }}$ dimension and $z$ is the space dimension.

Step 2: Fitness evolution and best fitness computation update best and worst: The fitness evolution is performed by evaluating the best and worst fitness for all agents at each iteration, for minimization or maximization problems,

Maximization problems:

$\operatorname{Best}(q)=\min \operatorname{fit}_{k}(q), \quad k \in\{1, \ldots, \ldots . Z\}$

$\operatorname{Worst}(q)=\max \operatorname{fit}_{k}(q), \quad k \in\{1, \ldots, \ldots Z\}$

Minimization problems:

$\operatorname{Best}(q)=\min$ fit $_{k}(q), \quad k \in\{1, \ldots, \ldots . Z\}$

$\operatorname{Worst}(q)=\max \operatorname{fit}_{k}(q), \quad k \in\{1, \ldots, \ldots . Z\}$

$\operatorname{fit}_{k}(q)$ Represents the fitness value of the $k^{\text {th }}$ agent at iteration $q, \operatorname{Best}(q)$ and $\operatorname{Worst}(q)$

Represents the best and worst fitness at iteration q. 
Step 3: Update Gravitational constant $(G)$ computation: Gravitational constant $G$ is computed at iteration $\mathrm{q}$.

$$
G(q)=G_{0} e^{\left(\frac{-\beta q}{T}\right)}
$$

$G_{0}$ And $\beta$ are initialized at the beginning and will be reduced with time to control the search accuracy. $\mathrm{T}$ is the total number of iterations.

Step 4: Calculate masses and Acceleration of the agents: Gravitational and inertia masses for each agent are calculated at iteration q.

$$
\begin{aligned}
& M_{x l}=M_{y l}=M_{l i}=M_{i} \text { For } i=1 \ldots, 2 \ldots, Z \\
& m_{l}(q)=\frac{\operatorname{fit}_{l}(q)-\operatorname{worst}(q)}{\operatorname{best}(q)-\operatorname{worst}(q)} \\
& M_{i}(q)=\frac{m_{l}(q)}{\sum_{k=1}^{Z} m_{j}(q)}
\end{aligned}
$$

$M_{x l}$ and $M_{y l}$ are active and passive gravitational masses respectively, $M_{l i}$ is the inertia mass of the $l^{\text {th }}$ agent.

Calculate acceleration of the agents:

Acceleration of the $k^{\text {th }}$ agents at iteration $q$ is computed.

$a_{k}^{d}=F_{k}^{d}(q) / M_{l k}(q)$

$F_{k}^{d}(q)$ is the total force acting on $k^{\text {th }}$ agent calculated as:

$F_{k}^{d}(q)=\sum_{s \in u b e s t, s \neq k} \operatorname{rand}_{s} F_{k s}^{d}(q)$

Step 5: Update Position and velocity using PSO: In PSO, $p_{i}^{d}(q)$ and $v_{i}^{d}(q)$ are calculated as follows

$$
\begin{aligned}
& p_{i}^{d}(q)=p_{i}^{d}(q)+p_{i}^{d}(q+1) \\
& \left.v_{i}^{d}(q+1)=W(q) v_{i}^{d}(q)+F_{1} R_{k 1}\left(\text { Pbest }_{i}^{d}-p_{i}^{d}(q)\right)+F_{2} R_{k 1} \text { Sbest }_{i}^{d}-p_{i}^{d}(q)\right)
\end{aligned}
$$

Where, $R_{k 1}$ and $R_{k 1}$ are two random variables in the range [0,1], $F_{1}$ and $F_{2}$ are positive constants, $W$ is the inertia weight. $p_{i}$ and $v_{i}$ represents position and velocity of the $i^{\text {th }}$ agent respectively.

Step 6: Criteria check: Steps 2 to 5 are repeated until the iterations reach their maximum limit $\mathrm{T}$. The best fitness value at the final iteration is computed as the global fitness while the Position of the corresponding agent at specified dimensions is computed as the global solution of that particular problem.

\section{Result and Discussion}

The main aim of the paper is to minimize the critical issue of WSN, that is energy consumption and hops in the routing choice. In this section, proposed technique and existing techniques will be implemented on working plate form of MATLAB to investigate the performance of proposed technique. The comparative analysis will be jotted, based on that conclusion will be framed. 
Energy Dissipation: The energy of nodes is dependent on the power which is embedded in nodes, so we always have the limitation for the cost and the size of the nodes. AGSA algorithm is a new method for reducing the energy consumption in the nodes routing. The energy dissipation for nodes employing different optimization techniques is depicted in figure 3.

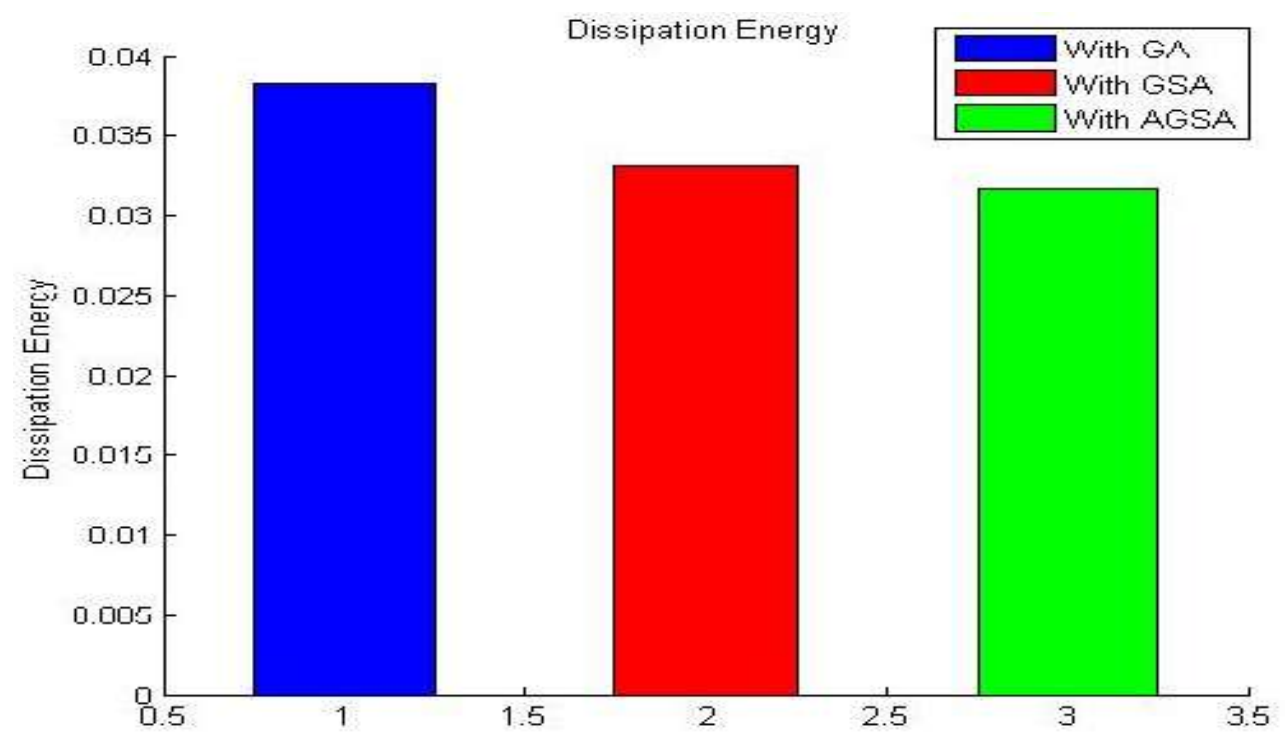

\section{Figure 3. Dissipation Energy}

In the above figure, magnitude of energy dissipation of two optimizations (GA and GSA) other than proposed technique (AGSA) is depicted. It can be noted from the figure that, energy dissipated by the node employing AGSA optimization is less than GA and GSA. We can say that AGSA has improved the performance of energy dissipation. This dissipation could have been in the form of heat or in any other form.

Direct distance: The AGSA optimization employs Closest forwarding (CF) scheme. In the Closest Forwarding (CF) scheme, a node only forwards its traffic to its closest neighbor toward the data sink. The CF scheme consumes the least overall energy. We can imagine this scheme to be operating in a network where all nodes are location nearby to each other. For GA, GSA and proposed technique AGSA, direct distance estimation is given in figure 4. 


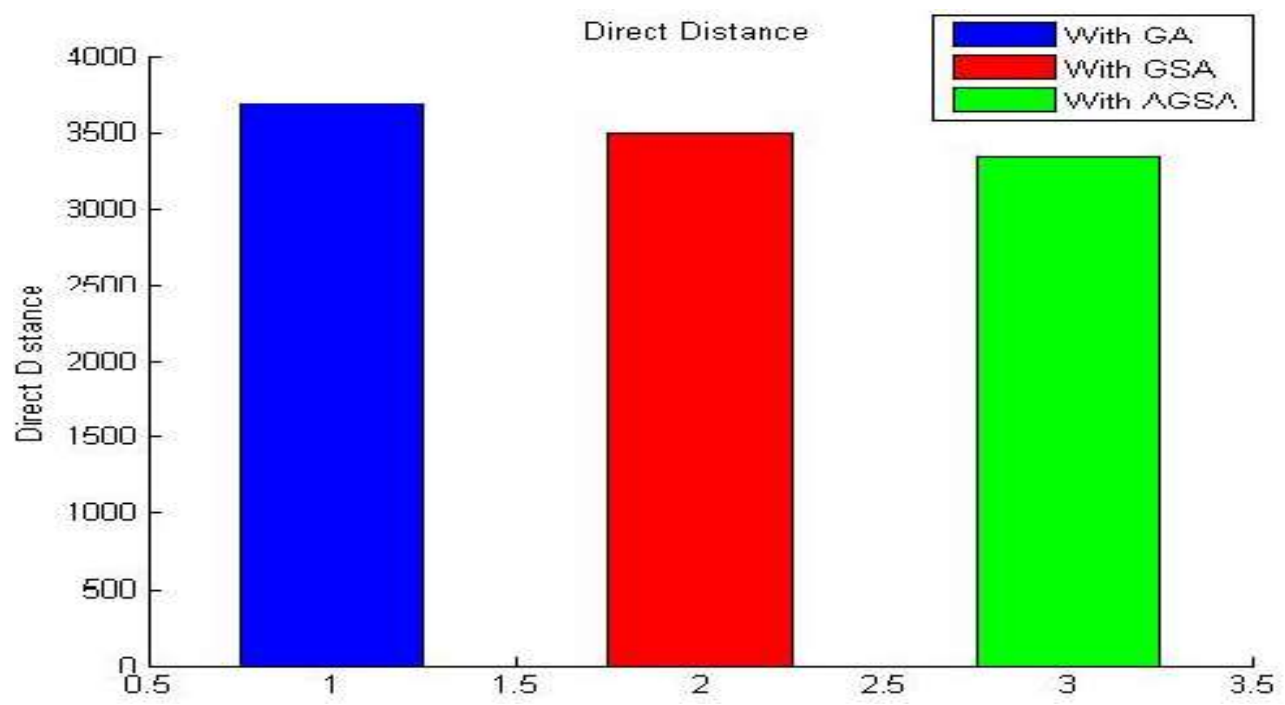

Figure 4. Direct Distance

It can be noticed from the above figure that the proposed optimized technique has improved performance for short distance forwarding of frames. Due to the improvement least energy is consumed by the node.

Compactness: It can be noted from the above figure that, the proposed optimization techniques (AGSA) has improved the performance of compactness. AGSA has less compactness as compared to the existing techniques, as shown GA and GSA.

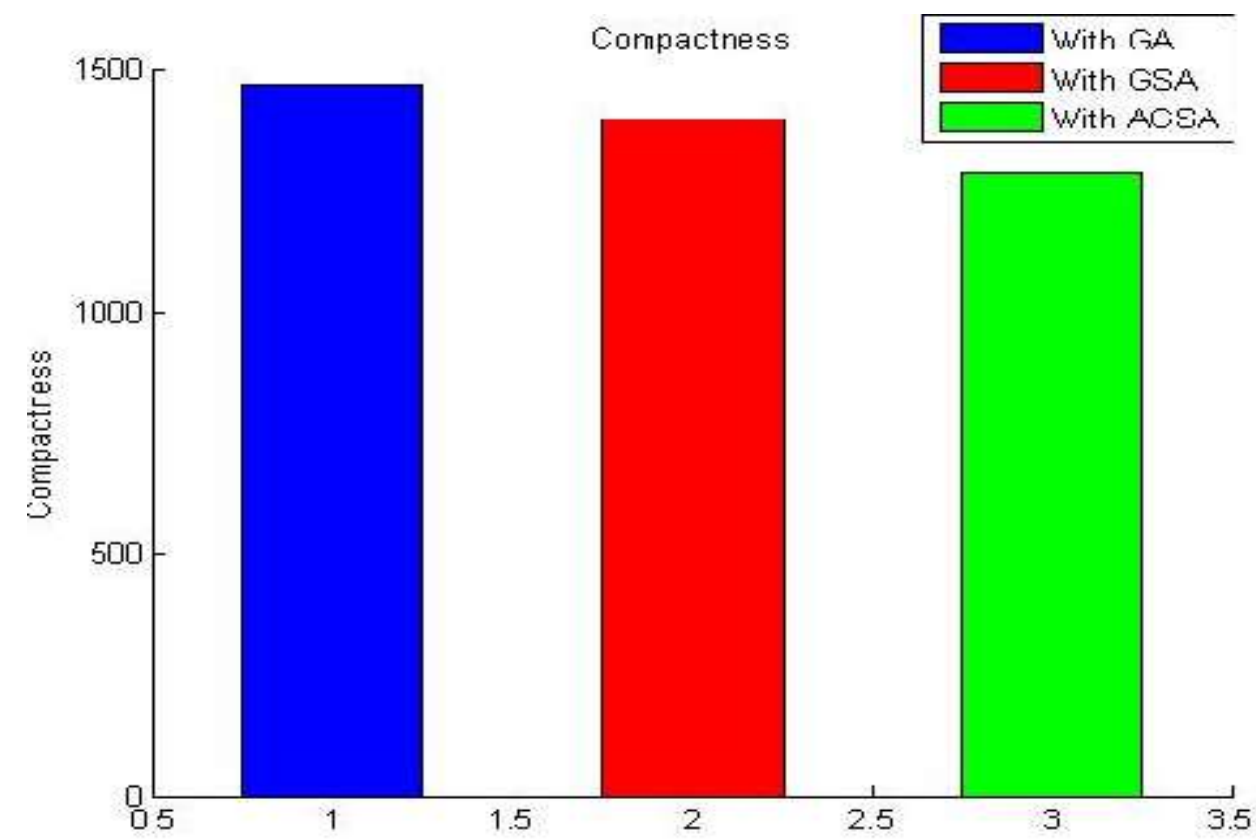

Figure 5. Compactness

Total Energy: The calculation of the energy consumed by the individual sensor nodes or the total energy consumption of the sensor network for different optimization techniques is depicted below in the figure 6 . 


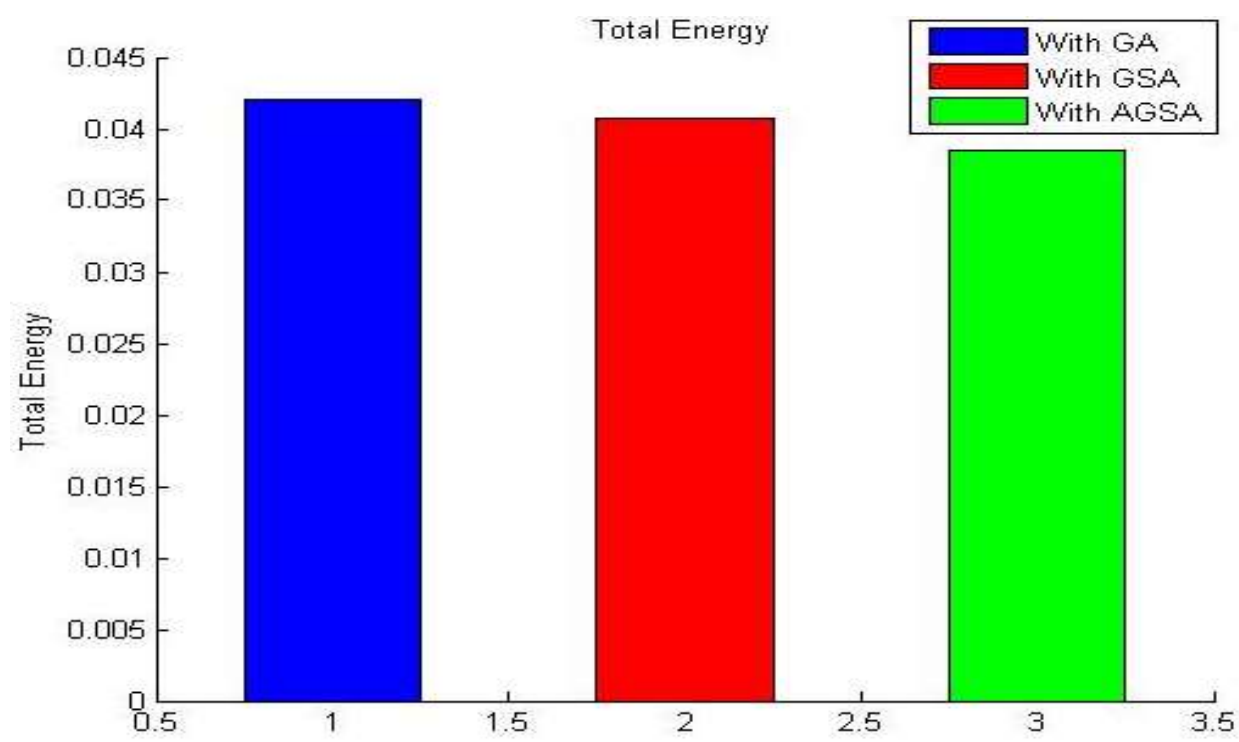

Figure 6. Total Energy of Sensor Network

It can be noted from the above figure that, the proposed optimization techniques (AGSA) overall consumes less energy as compared to the existing techniques, as shown GA and GSA.

\section{Conclusion}

Adaptive Gravitational Search Algorithm (AGSA) based routing techniques have been implemented for power consumption reduction. The proposed method (AGSA) showed better performance as compared to the existing techniques. In this paper we have achieved success in improving the battery life of the node or the sensor network, which can be noted from the Results section. In future, this work can be taken to the next level by eliminating certain assumptions of this paper, like we have assumed CF technique for packet forwarding to reduce power consumption. Secondly, advanced optimization can be employed for efficient routing.

\section{References}

[1] E. F. Nakamura, H. S. Ramos, L. A. Villas, H. A.B.F. de Oliveira, A. L.L. de Aquino and A. A.F. Loureiro, "A reactive role assignment for data routing in event-based wireless sensor networks", Computer Networks, vol. 53, no. 12, (2009), pp. 1980-1996.

[2] A. Ahmed, K. Abu Bakar, M. I. Channa, A. W. Khan and K. Haseeb, "Energy-aware and secure routing with trust for disaster response wireless sensor network", Peer-to-Peer Networking and Applications, (2015).

[3] E. A. Khalil and B. A. Attea, "Stable-Aware Evolutionary Routing Protocol for Wireless Sensor Networks", Wireless PersCommun, vol. 69, no. 4, (2012), pp. 1799-1817.

[4] W. Ke, Y. Ou, H. Ji, H. Zhang and L. Xi, "Energy aware hierarchical cluster-based routing protocol for WSNs", The Journal of China Universities of Posts and Telecommunications, vol. 23, no. 4, (2016), pp. 46-52.

[5] A. A. abba Ari, B. Omer. Yenke, Nabila Labraoui, Ireprandamakoa and Abdelhakgueroui, "A power efficient cluster-based routing algorithm for wireless sensor networks: Honeybees swarm intelligence based approach", Journal of Network and Computer Applications, vol. 69, (2016), pp. 77-97.

[6] P. Kuila and P. K. Jana, "Energy efficient clustering and routing algorithms for wireless sensor networks: Particle swarm optimization approach", Engineering Applications of Artificial Intelligence, vol. 33, (2014), pp. 127-140.

[7] NaércioMagaia, NunoHorta, RuiNeves, Paulo Rogério Pereira and Miguel Correia, "A multiobjective routing algorithm for Wireless Multimedia Sensor Networks", Applied Soft Computing, vol. 30, (2015), pp. 104-112. 
[8] S. Maryam and N. H. Reza, "A decentralized energy efficient hierarchical cluster-based routing algorithm for wireless sensor networks", AEU - International Journal of Electronics and Communications, vol. 69, no. 5, (2015), pp. 790-799.

[9] H. Shen and G. Bai, "Routing in wireless multimedia sensor networks: A survey and challenges ahead", Journal of Network and Computer Applications, vol. 71, (2016), pp. 30-49.

[10] M. Yigit, V .C. Gungor, E. Fadel, L. Nassef, N. Akkari and I. F. Akyldiz, "Channel-aware routing and priority-aware multi-channel scheduling for WSN-based smart grid applications", Journal of Network and Computer Applications, vol. 71, (2016), pp. 50-58.

[11] M. Jahanshahi, M. Dehghan and M.R. Meybodi, "A mathematical formulation for joint channel assignment and multicast routing in multi-channel multi-radio wireless mesh networks", Journal of Network and Computer Applications, vol. 34, no. 6, (2011), pp. 1869-1882.

[12] X.-C. Hao, X.-Y. Ru, X.-D. Liand, M.-J. Xin, "Energy Efficient Based Channel Assignment Game Algorithm for Wireless Sensor Network", Wireless PersCommun, vol. 85, no. 4, (2015), pp. 2749-2771.

[13] J. Li, X. Guo, L. Guo, S. Ji, M. Han and Z. Cai, "Optimal routing with scheduling and channel assignment in multi-power multi-radio wireless sensor networks", Ad Hoc Networks, vol. 31, (2015), pp. 45-62.

[14] Sok-Hyong Kim, Dong-WookKimaand Young-JooSuh, , "A group-based channel assignment protocol for rate separation in IEEE 802.11-based multi-radio multi-rate ad hoc networks", Ad Hoc Networks, vol. 10 , no. 1, (2012), pp. 95-110.

[15] H. A. Mogaibel, M. Othman, S. Subramaniam and N.A.W. Abdul Hamid, "Review of channel assignment approaches in multi-radio multi-channel wireless mesh network", Journal of Network and Computer Applications, vol. 72, (2016), pp. 113-139. 